\title{
La vie religieuse en mer
}

Jaime Alvar et Mirella Romero Recio

Madrid

La mer est un réservoir inépuisable d'inspiration pour l'expression des phénomènes surnaturels. Probablement parce que les vicissitudes de ceux qui en vivent et qui y habitent sont souvent extrêmes. Le recours à des explications invraisemblables et le désir de s'attirer les volontés divines sont finalement inévitables pour survivre dans un milieu d'une hostilité manifeste.

L'aversion envers la mer parmi ceux qui en vivent est fort ancienne. Caro Baroja a exploré les origines de la piélagophobie ${ }^{1}$. Apparemment, elle s'articule autour de deux idées de base relatives à l'altération de l'ordre naturel. D'un côté, la modification arbitraire de la Nature comme conséquence de l'exploitation de ses ressources. D'un autre, l'appât du gain. Ces deux altérations sont la conséquence de l'hybris et elles entraînent donc un châtiment, qui doit être compris comme une juste compensation dans les va-et-vient de l'existence. Nombreux sont les exemples en ce qui concerne la première idée, mais Pline nous en fournit un de paradigmatique. Après avoir considéré que l'évolution des techniques de navigation est la preuve du développement et du progrès de l'Humanité ( $\mathrm{NH}$. VII, 56-57), il affirmera plus loin sans problème que les inventions navales sont un grand mal et, au premier chef, la voile. La découverte du filage et du tissage a permis leur application à la navigation, comme l'indique Pline en parlant du lin $(N H$. XIX, 1). Mais cette violence faite à l'ordre naturel était une véritable injure pour la Nature, à cause de quoi Icare, l'inventeur de la voile, fut l'objet de la plus terrible des malédictions puisqu'il mourut en mer sans sépulture².

J. Caro Baroja, De la superstición al ateísmo (Meditaciones antropológicas), Madrid, 1974, 67 sqq.

2 Au sujet de la tragédie qui accompagne la mort en mer et l'absence de sépulture : J.-P. Vernant, "La belle mort et le cadavre outragé", in G. Gnoli, J.-P. Vernant (dirs), La mort, les morts dans les sociétés anciennes, Cambridge, 1990 (1982), 68 ; S. Georgoudi, "La mer, la mort et le discours des épigrammes funéraires", AION (archeol), 10, 1988, 53-61 ; M. Romero Recio, "Conflictos entre la religiosidad familiar y la experiencia sacra de los navegantes griegos", ARYS, 1, 1998, 43-46. 
Dans ce même ordre d'idées au sujet de l'altération de la Nature en tant que source de maux, Ovide indique qu'à l'Âge d'Or “le pin n'était pas coupé à la hache dans la montagne et il n'était pas descendu jusqu'à la plaine liquide, pour visiter des terres étrangères. Les hommes ne connaissaient que les choses naturelles (Mét. I, 95-96). C'est lorsque les crimes dominent le monde que le navigateur s'abandonne aux vents avec la voile. Les arbres descendus des sommets, défient et insultent l'onde (Mét. I, 132-134)"3.

La deuxième idée qui participe de la piélagophobie est celle de l'appât du gain. Le rejet de la démesure est lui aussi fort ancien, puisque Hésiode lui-même, vers le VII ${ }^{\mathrm{e}}$ siècle, s'exprimera clairement à ce sujet. Personne ne doit se risquer en dehors de la saison nautique et silloner la mer à la recherche de bénéfices extraordinaires (Erga 684-686), car la misère est un don funeste de Zeus (Erga 638). Les naufrages sont la conséquence du risque assumé, provoquant la moins désirée des morts puisque les cadavres ne peuvent avoir de sépulture. Nombre des épigrammes du livre IX de l'Anthologie Palatine constituent en ce sens une collection de textes impressionnante. Mais ce n'est pas uniquement là que l'on peut trouver ce que Grecs et Romains ressentaient par rapport à tout ceci. Palinurus, le pilote de la flotte d'Énée, ne trouve le repos que lorsque le héros troyen vient le voir dans l'Hadès. Énée l'enterrera et il cessera d'errer comme tant d'autres capitaines naufragés (Énéide VI, 333-381; au sujet de la mort de Palinurus, V, 840-861). D'après la légende, le cap Palinurus, près de Vélia, s'appelait ainsi en son honneur, et le héros fut objet de vénération dans cette zone4. L'impiété que suppose la navigation finit par devenir un topos dans la poésie latine et Horace en est le représentant le plus remarquable (Carm. I, 1, 15-18 ; II, 16, 1-4 ; 21-24 ; Sat. I, 1, 6-7). Avec lui nous trouvons Properce lorsqu'il parle, par exemple, de l'avare Péton (Eleg. III, 7), Juvénal (Sat. 12, 57-59; au sujet de l'avare qui surcharge son vaisseau dans Sat. 14, 288-302) et Ovide lui-même écrit que ce qui l'effrayait le plus dans son voyage à Tomis était de mourir noyé (Trist. I, 2, 51-56). Ovide pouvait solliciter la protection divine car la raison de son voyage n'était pas liée au profit. Neptune est le dieu qui châtie ceux qui se sont enrichis en mer sans

3 Caro Baroja, De la superstición al ateísmo..., 68

4 Cf. Virg. Aen. 3.200-204, 269, 513-520, 561-563 ; 5.12-25 ; D.H. 1.53 .2 ; D.C. 49.1-2 ; Vell. 2.79.3-4 ; Oros. 4.9.11; Serv. ad Aen. 6.378-379. A.G. McKay, "Vergilian Heroes and Toponymy : Palinurus and Misenus", in Mnemai, Classical Studies in Memory of K.K. Hulley, Evjen, H.D. (éd.), California, 1984, 130-137 ; W.S.M. Nicoll, W.S.M., “The sacrifice of Palinurus”, CQ, 38, 1988, 459-472. 
vergogne (Plaute, Rudens, Acte II, scène III, 371-372) raison pour laquelle tous les naufragés sont soupçonnés de conduites répréhensibles5.

Il est curieux d'observer la persistance au fil du temps de cette vision de la mer, à tel point qu'au XVI ${ }^{\mathrm{e}}$ siècle, dans les grands empires maritimes, apparaissent des auteurs qui rejettent les entreprises maritimes car elles continuent de représenter l'avarice, la corruption et le luxe face à la vie austère des paysans et des soldats, fondement des modes de vie traditionnels ${ }^{6}$. La piélagophobie atteint son point culminant dans le livre de Bernardo Gómes de Brito, publié entre 1735 et 1736 sous le titre de Historia trágico-marítima. Deux volumes qui recueillent les plus impressionants récits de naufrages, où la surcharge est un élément présent à plusieurs reprises 7 .

Cependant, notre érudit ethno-anthropologue ne prit pas en considération un troisième facteur qui provoque la piélagophobie, basé sur le pouvoir cathartique de l'eau. Les documents ne manquent pas sur la fonction de la mer comme destinataire de maux physiques et spirituels. D'ailleurs, "l'expulsion des maux vers la mer" est le titre d'un des épigraphes du très beau livre de Pedrosa où il analyse la présence de cet élément dans le folklore de différentes traditions 8 . C'est probablement dans l'Iphigénie d'Euripide que nous trouvons l'un des exemples les plus anciens de cette capacité cathartique, lorsque Iphigénie affirme que "la mer lave tous les maux des hommes" 9 . Une fois que la pollution est passée dans ce milieu il reste maudit ${ }^{10}$. De là, il est dépositaire d'immondices er demeure de monstres déjà depuis la tradition biblique ${ }^{11}$. Cependant, comme le rappelle Pedrosa lui-même, "les rites d'expulsion de maux vers les courants d'eau sont une manifestation particulière de la typologie - beaucoup plus vaste - des croyances et des pratiques d'expulsion de maladies, tempêtes et esprits malins

\footnotetext{
5 Le naufragé est considéré comme un homme abandonné des dieux. Cf. Romero Recio, "Conflictos entre la religiosidad familiar...", 43.

6 Caro Baroja, De la superstición al ateísmo..., 73.

7 Voir l'édition réalisée par Rodrigues Lapa à Lisbonne en 1956 sous le titre Cuadros da história trágico-marítima que l'érudition de Caro Baroja nous a fait connaître (De la superstición al ateísmo..., 74).

8 J.M. Pedrosa, Entre la magia y la religión : oraciones, conjuros, ensalmos, Oiartzun, 2000, 92-98.

9 Eurípides, Ifigenia entre los tauros, in Tragedias II, 3 vol., J.L. Calvo Martínez (éd.), Madrid BCG, 1978, 396.

10 E.E. Burriss réalisa un intéressant recueil de rites de transmission des maladies vers l'eau dans les sociétés primitives dans Taboo, magic, spirits : a study of primitive elements in Roman religion (rééd. Westport, Connecticut, Greenwood, 1972, pp. 150-153), repris, naturellement, dans le livre très documenté de Pedrosa.

11 Pedrosa, Entre la magia y la religión..., 96, note 50.
} 
vers des lieux très divers ... puits, arbres, cimetières ou carrefours ... chiens, ânes, grenouilles ... passants, morts"12.

Ainsi, le transfert des maux vers la mer a de même contribué de manière solide et vaste à la perception négative de la mer et au développement de l'imaginaire "piélagophobique". Il n'est donc pas surprenant que la mer fournisse une très riche information de caractère religieux sur les comportements rituels ou magiques qui en découlent.

Naturellement, la mer et les activités qui s'y développent sont à l'origine d'une image éthique, mais en même temps, de croyances spécifiques qui s'accroissent et se transforment au fur et à mesure que les expériences en mer et les craintes qui en surgissent, augmentent. C'est ainsi que les gens de la mer inventent des histoires au sujet des phénomènes incompréhensibles, vécus ou imaginés, qu'ils ritualisent les rythmes de leurs travaux et les identifient avec des messages envoyés par les dieux et qu'ils multiplient en plus le nombre d'entités surhumaines pour s'assurer de leur bon vouloir et, par là, d'une heureuse survie.

Tout cela fait qu'il n'est pas possible d'aborder en quelques pages la totalité des aspects susceptibles d'être étudiés, sous le seul titre de "La vie religieuse en mer". Nous nous en tiendrons donc à quelques aspects qui mettent en relief la versatilité de la religiosité des navigateurs et son rapport avec la vie religieuse des cités.

Bien que les gens de la mer connurent des manifestations religieuses de caractère spécifique, il est important de rappeler qu'ils étaient membres de communautés qui possédaient un système religieux complet et que l'activité maritime n'était qu'une des multiples branches de leur économie. Nous ne pouvons donc pas parler de la religion des navigateurs car ce serait aussi peu approprié que de parler de la religion des paysans ou de la religion domestique. En réalité, il ne s'agit que d'expressions cultuelles parmi toutes celles d'un système plus vaste qui est celui de la religion de la communauté à laquelle ils appartiennent tous. Sans doute, la cité participe aux cultes maritimes de la même façon que les marins participent au reste des cultes civiques. Chaque chose a lieu en son temps, c'est-à-dire, au moment qui convient le plus à sa réalisation. Par conséquent, c'est des cultes maritimes, des cultes agraires, des cultes militaires ou des cultes domestiques à l'intérieur de la religion de l'État, qu'il faut parler.

12 Pedrosa, Entre la magia y la religión..., 98, note 53. 
La mer des Anciens était pleine de monstres, d'êtres hybrides qui survécurent jusqu'au Moyen Âge et qui vivent encore dans quelques replis sombres de notre cerveau lorsque nous gardons l'espoir d'apercevoir le monstre du Loch Ness ou que nous voulons voir des calmars géants, dont l'invraisemblable apparence tient lieu d'explication supposée logique pour les croyances de nos ancêtres. Dans notre perversion cartésienne nous voulons expliquer que nos ancêtres, après avoir aperçu l'un de ces rares animaux des abysses, recréèrent à partir d'eux des histoires de tritons et de néréides ou alors, ayant aperçu le saut d'un dauphin, ils imaginèrent une belle sirène incapable de leur procurer la satisfaction tant souhaitée. Ou même que, oublieuses devant la cithare d'Orphée, elles seraient capables de faire échouer la traversée du navigateur.

Caro Baroja pense que quelques traditions de la péninsule concernant des êtres marins trouvent peut-être leur origine dans l'Antiquité elle-même et il rappelle à ce propos le texte de Pline où est mentionnée l'existence d'un homme marin dans nos eaux juridictionnelles. Pour écarter tous les doutes possibles, Pline s'appuie sur le témoignage de quelques membres de l'ordre équestre qui auraient vu monter sur leur bateau un marinum hominem une nuit de tempête sur l'Océan gaditain (N.H. IX, 5, 10). Depuis lors, l'apparition de tritons et de néréides devient de plus en plus fréquente, à tel point qu'elle force la crédulité de beaucoup de personnes, parmi lesquelles un esprit aussi éclairé que celui du philosophe espagnol le plus important du XVIII e siècle, l'abbé Feijóo'13. La croyance en l'existence de tels êtres avait été si intense que l'abbé Fuentelapeña, dans son Ente dilucidado de 1676, nous fait savoir ce qui suit: "Qu'il y ait de tels Tritons n'est pas à mettre en doute, comme il en ressort des archives du Portugal : où il y eut autrefois procès entre le Roi et le Maître de Santiago, sur à qui revenait le Tribu des Tritons et Sirènes de la mer, et la sentence fut que le Tribu des Sirènes revenait au Roi, et celle des Tritons au grand Maître"14. Quoi qu'il en soit, Feijóo crut à l'histoire de l'homme poisson de Lierganès :

"Le jour de la veille de la Saint Jean de 1673, Francisco de la Vega Casar, habitant de Lierganès, Santander, partit se baigner avec d'autres garçons dans l'estuaire de Bilbao, localité où il était en train d'apprendre un métier. Il se jeta à la mer et ne reparut plus. Les camarades réputèrent qu'il s'était noyé.

13 Teatro crítico universal, tome VI, Madrid, 1771, 314-317.

14 El ente dilucidado. Discurso único novísimo que muestra hay en naturaleza animales irracinales invisibles, y cuales sean p. 187 (num. 705), cité par J.Caro Baroja, Algunos mitos españoles. Ensayos de mitología popular, Madrid, 1941, p. 154. 
En 1679 des pêcheurs gaditains virent au milieu de la mer, nageant avec une grande habileté, "une figure de personne rationnelle", qu'ils purent capturer après plusieurs tentatives, dont il résulta qu'il s'agissait dudit Francisco, lequel, une fois revenu chez lui, vécut neuf ans de façon extravagante, pour disparaître ensuite sans laisser de traces $» 15$.

Feijóo invoque des exemples parallèles plus ou moins proches pour justifier la vraisemblance de l'histoire, mais ce qui a été dit sur la capacité d'invention des gens de la mer et sur la crédulité de leurs compatriotes suffit à montrer que la mer est si grande qu'elle peut être le lieu de tous les fantasmes. Ainsi Pausanias croit-il à l'histoire selon laquelle des navigateurs auraient découvert des îles dans la mer Extérieure lorsqu'ils furent déviés vers l'Italie. Il s'agissait des îles Satyriades où habitaient des êtres étranges dorés par le soleil et qui avaient une queue semblable à celle des chevaux. Quand le vaisseau arriva sur ces parages, les habitants se lancèrent sur le bateau pour prendre les femmes qu'il y avait à bord. Les marins effrayés leur donnèrent pour les calmer une femme barbare qu'ils outragèrent "non seulement par où il est convenu, mais aussi par tout le corps" (I, 23, 5-6) ${ }^{16}$.

Depuis la plus haute Antiquité la mer était peuplée non seulement d'êtres hybrides intermédiaires mais aussi de multitudes de dieux et de daimônes, de mythes et de légendes. Le développement des thalassocraties, c'est-à-dire des États qui fondaient leur pouvoir économique sur la domination de la mer, entraîna la régulation des rapports entre les hommes de la mer et les dieux maritimes, car les uns et les autres se devaient d'agir au bénéfice de la religion civique. La réflexion que nous soutenions au début, selon laquelle la religion de la mer fait partie de la religion de la cité, prend par là tout son sens.

Et c'est ainsi que les dieux de la mer vont posséder leurs propres sanctuaires dans des enclaves significatives dispersées sur tout le littoral méditerranéen, nombre d'entre eux en des lieux adéquats pour calculer les distances, d'autres sur des lieux particulièrement difficiles pour la navigation et, par là, demeure d'êtres malins à la physionomie impossible et friands de naufragés. Héra devient la protectrice des ports sous le nom de "Liménia", en guise de quoi on lui consacre le fameux sanctuaire de Pérachora ; Artémis est la protectrice des navigateurs, de là

15 Caro Baroja, Algunos mitos, p. 149-150.

16 Sur les représentations possibles de cette scène dans la céramique : M. Torelli, "L'immaginario greco dell'oltremare. La lekythos eponima del Pittore della Megera, Pausania I, 23, 5-6 e Pitecusa" in: APOIKIA, Scritti in onore di Giorgio Buchner, Annali di Archeologia e Storia Antica, N.S. 1, D'Agostino, B., Ridgway, D. (éds), Naples, 1994, 117-125. 
elle eut un temple sur un lieu dangereux de la côte eubéenne ; au Cap Sounion, $c^{\prime}$ est Poséidon que les navigateurs invoquent, et ainsi de suite ${ }^{17}$.

Artémis est une déesse très impliquée dans la navigation. Dans l'Iliade déjà, elle se manifeste capable de s'opposer à l'attaque mycénienne qui a pour mission d'en finir avec l'amour entre Hélène et Pâris. Pour cela, elle envoie des vents contraires à ceux sollicités par l'indésirable Agamemnon. À Aulis, où se trouvait réunie la flotte coalisée, le chef achéen sait que la seule solution qui lui reste est d'amener sa fille Iphigénie sur l'autel du sacrifice. Artémis intervient de nouveau, conduisant la victime saine et sauve en Tauride (Crimée) ${ }^{18}$. On a souvent discuté pour savoir si cet épisode reflétait l'existence de sacrifices humains en Grèce. Il y en eut sans doute, mais il est difficile d'admettre qu'à l'époque historique ils furent toujours nombreux même si les tempêtes menaçaient une embarcation ou les intérêts d'un État. D'autre part, la colonisation grecque de la Méditérranée, en particulier dans sa moitié occidentale, fut pour l'essentiel menée à bien par des Grecs Ioniens. La déesse tutélaire d'Éphèse était une Artémis très spéciale, car elle avait conservé sur sa physionomie des traits archaïques en rapport avec la fertilité et l'alimentation, c'est pourquoi tout son corps est recouvert de seins et elle a comme épithète "Polymastia". Cette Artémis éphésienne se répandit sur toute la Méditérranée et de nombreuses expéditions coloniales s'effectuèrent sous son patronage. Avec elle, le dieu masculin le plus important dans la colonisation fut, évidemment, Apollon, qui sous divers vocables est le protecteur des navigateurs, de leurs bateaux et des territoires explorés et fréquemment intégrés dans l'Hellade (voir par exemple, Apol. Rod. Arg. I, 360 et 404, où l'on nomme Apollon Embasioio, c'est-à-dire, dieu de l'embarquement, qui à travers ses oracles montre les routes à naviguer; mais il l'est aussi du débarquement : Ekbasioo, ibidem, 966 ; objet de sacrifices particuliers, ibidem, 11851186).

Par contre, Athéna favorisa Jason et contribua à résoudre la navigation sur une mer Noire alors inconnue, en fournissant une table parlante à travers laquelle la déesse s'exprimait; à l'opposé, on peut mentionner l'intervention constante de Poséidon pour empêcher Ulysse de revenir auprès de Pénélope,

17 M. Romero Recio, in D. Plácido et alii (éds), "Limén y espacio sacro en el mundo colonial", in Imágenes de la polis. Madrid, 1997, 389-406; eadem, Cultos marítimos y religiosidad de navegantes en el mundo griego antiguo, BAR 897, Oxford, 2000, 113 sqq.

18 A. Ag. 1256 ; E. IT, 354-358 ; Or. 23 ; Apollod. epit. 2.16 ; 3.21 ss ; Ov. met. 12.24-38 ; Hyg. fab. 98, 120, 238, 261 ; Nonn. 13.107 ; Procl. Chr. 1. Cf. Paus. 2.22.6-7 ; Ant. Lib. 27. Sur les différentes versions du mythe voir: P. Bonnechere, Le sacrifice humain en Grèce ancienne (Kernos suppl. 3), Athènes-Liège, 1994, 26 sqq. 
mais ses pouvoirs sont contrecarrés par Athéna, assimilée parfois à la déesse Leucothée, qui le sauve dans le naufrage qui constitue l'une des scènes les plus dramatiques de l'Odyssée (v. 332 sqq.), en lui prêtant son voile grâce auquel il atteint le rivage et d'où il le rend à la mer, tel qu'on le lui avait indiqué. Comme déesse de la techne, Athéna est inventrice et c'est à elle que l'on attribue la construction d'importants vaisseaux dans le cycle mythologique grec.

En tant que dieu suprême, Zeus est de même responsable du calme marin. Comme protecteur des navigateurs il reçoit l'épithète de "Soter" ; comme avertisseur des lieux dangereux celui de "Kassios", et ainsi de suite.

À l'époque romaine on remarque le culte marin des Dioscures, Castor et Pollux, les frères jumeaux fils de Zeus et de Léda. Leur constellation était l'une des plus utilisées par les navigateurs dans les traversées nocturnes. Ils se présentent aux marins sous leur aspect le plus saisissant dans le feu de Saint-Elme, qui surgit comme conséquence de la surcharge électrique dans l'atmosphère et qui se manifeste tout au bout du mât ou des vergues. L'équipage sait alors qu'il est à l'abri, puisque les dieux ont mis fin à la tempête et se sont installés dans le vaisseau pour le mener à bon port.

Ces dieux qui interviennent de façon si active dans la vie des marins font l'objet d'une attention constante. On leur offre des sacrifices avant d'entreprendre la traversée: dans les Argonautiques orphiques (333-357) les héros sacrifient un taureau dont le sang est mélangé à de la farine d'orge et à de l'eau de mer, pour invoquer ensuite l'aide de tous les dieux marins avant d'appareiller, les héros se purifiant ainsi de l'intérieur (face à l'offrande de l'eau versée sur la coque du navire), et ils partagent avec les dieux le sang de la victime qui doit leur procurer le salut auquel contribue l'orge de Déméter. Il est même fréquent que tout l'équipage se dirige en procession vers le sanctuaire le plus proche pour réaliser une offrande. Dans les fameuses Lois de Gortyne on trouve une clause se rapportant à la purification des vaisseaux avant de quitter le port (Inscr. Cret. IV, 146, 1.1 ; libations avant de partir à Céphisodore ap. Poll. X, 109); Thucydide (VI, 32) indique comment avant d'appareiller et une fois les vaisseaux équipés, on donnait le signal du silence et on faisait les prières traditionnelles. Tous les bateaux mélangeaient en même temps le vin dans des cratères et faisaient des libations dans des vases en or et en argent. Une fois en mer, le capitaine effectue un sacrifice et prononce des prières au dieu tutélaire du vaisseau lorsqu'on atteint la pleine mer. Ensuite, il réalise des libations ou des sacrifices en passant devant d'importants sanctuaires côtiers et dans les espaces sacrés situés sur d'éminents accidents géographiques. On implore aussi les dieux dans des circonstances 
adverses et, avant de rentrer au port, on renouvelle les sacrifices et les offrandes en signe de remerciement. La Silva III $(2,21-24)$ de Stace nous apprend que lorsque le convoi arrivant d'Égypte avec le blé nécessaire pour nourrir les habitants de Rome atteignait l'île de Capri, on réalisait le sacrifice d'arrivage qui consistait en une libation de vin en honneur de Minerve. Finalement, une fois le bateau déchargé, on se dirige de nouveau vers le sanctuaire du port pour tenir les promesses et déposer la contribution pécuniaire correspondante. Les ex-votos en rapport avec la navigation sont très nombreux et constituent le témoignage fidèle du soin que prenaient les navigateurs pour s'attirer les bonnes grâces des dieux ${ }^{19}$.

Pour mener à bien tous ces rites, les vaisseaux étaient préparés: ils portaient les victimes adéquates, les offrandes précises, les liquides pour les libations et le feu indispensable aux sacrifices. Au cap Gelidonya, au sud de la Turquie, un bateau sombra au XII ${ }^{\mathrm{e}}$ siècle av. J.-C., probablement parce que le feu qu'il transportait à bord se propagea partout. L'épave récupérée du fond de la mer permet de savoir que pendant la navigation on élaborait les métaux que l'on transportait et que le même four servait certainement à cuisiner ou à réaliser les sacrifices pertinents. Une loi rhodienne interdit de fait aux passagers de cuire du poisson à bord du vaisseau, ce qui implique la possibilité d'avoir du feu20.

Longtemps après, pendant la Première Guerre Punique, le jour de la bataille de Drepanum, les poulets pour l'oracle que transportait le vaisseau de l'amiral Claudius Pulcher ne voulurent pas manger. Le consul ne fit aucun cas du sinistre augure et au lieu d'éviter la confrontation, il jeta les poulets à la mer pour qu'ils boivent, vu qu'ils ne voulaient pas manger. La défaite des Romains lors de cette bataille fut retentissante. Personne ne douterait alors de l'efficacité des rites ancestraux. Dans de nombreux cas on témoigne en plus de la présence de petits temples et d'autels sur le pont de poupe. La libation de vin était spécialement agréable aux dieux protecteurs en mer. On soignait de telles cérémonies jusqu'au moindre détail; nous savons de fait que dans le dernier voyage d'Auguste, l'équipage portait des vêtements blancs pour la réalisation des rites.

Offrir des ancres était très fréquent. Sostrate $d^{\prime}$ Égine en déposa une en pierre dans le sanctuaire d'Apollon à Gravisca, vers le VI siècle av. J.-C. et sur la pointe occidentale de la Péninsule, au cap Saint-Vincent, il existait peut-être un sanctuaire à l'air libre où on aurait célébré un rituel phénicien avec offrande d'ancres sur lesquelles les navigateurs venus ensuite auraient réalisé des

Toutes ces questions ont été analysées dans leur globalité par Romero Recio, Cultos marítimos...

20 W. Ashburner, The Rhodian Sea-Law, Oxford, 1909, II.10. 
libations. Le fondement de cette hypothèse s'établit à partir d'un texte de Strabon, le géographe de l'époque d'Auguste, qui décrit sommairement un rituel effectué par les navigateurs sur le Promontoire Sacré (III, 1, 4). Sur le lieu, d'après le témoignage qu'il emprunta à Artémidore d'Éphèse, lequel se trouvait sur la Péninsule vers l'an 100 av. J.-C., il y avait des pierres dispersées en groupes de trois ou quatre, et ceux qui arrivaient là-bas devaient faire des libations, selon une coutume ancestrale, pour ensuite les faire rouler en les changeant de position. Autant la célébration de sacrifices que l'accès de nuit au Promontoire étaient interdits, moment où il était occupé par les dieux. Ceux qui souhaitaient participer au rituel devaient camper dans un parage proche et se rendre au Promontoire Sacré pendant la journée, en portant avec soi l'eau nécessaire aux libations, puisqu'il n'y en avait pas dans la zone. Strabon indique qu'Artémidore avait comparé le Promontoire avec un navire car il était constitué de trois îlots: l'un d'entre eux ressemblait à l'éperon et les deux autres, qui avaient de bons mouillages, aux oreilles de proue. Aviénus, dans Ora maritima 217, ajoute que le Promontoire est consacré à Saturne, identifié au dieu phénicien Baal Hamon. L'endroit devait ressembler à d'autres sanctuaires cananéens, enceintes à l'air libre sans temple ni autel. Parfois on témoigne de l'existence de temples de type "tabernacle", c'est-à-dire, portables, et qui par conséquent ne laissent pratiquement pas de traces archéologiques. Les pierres que l'on retournait devaient être des ancres, comme nous l'apprennent Ougarit, Kition et Byblos, et sur elles devaient s'effectuer les libations; non seulement sur celles où on réalisait l'offrande, mais aussi sur celles qui s'étaient accumulées au fil du temps. Tel serait, donc, le "rite des pierres retournées"21.

Nombreux sont les exemples transmis par les sources au sujet des rites maritimes durant la période coloniale. Par des informations diverses nous savons que lors du processus de fondation des colonies, le feu sacré était transporté depuis la métropole jusqu'à l'apoikia, de façon à partager non seulement les mêmes dieux mais aussi l'essence de leurs sacrifices.

Le point culminant des voyages de commerce consistait en la remise d'une dîme au sanctuaire de la divinité la plus importante de la cité dont provenait l'embarcation. L'anecdote de Kolaios de Samos offrant un énorme chaudron en bronze au temple de Héra, en signe de remerciement pour le très fructueux voyage qui l'avait amené à découvrir l'imposant marché de Tartessos (Hdt. IV,

21 M. Romero Recio, "El rito de las piedras volteadas (Str. 3. 1. 4)", Arys, 2,1999, 69-82 ; eadem, Cultos marítimos ..., 30-34.

DHA supplément 1, 2005 
92), est bien connue. D'autres armateurs faisaient des dons sous forme de statues, de vaisseaux, et autres offrandes en tout genre. Les triomphes navals étaient souvent couronnés par la remise d'une partie du butin ou même avec les vaisseaux pris à l'ennemi. Cette coutume semble s'être imposée après le triomphe de Salamine en 480 av. J.-C., lorsque furent consacrées à Poséidon trois trirèmes phéniciennes (Hdt. VIII, 121). Cependant, la coutume est antérieure car Apollodore ${ }^{22}$, dans le récit du voyage des Argonautes, indique que Jason, après être retourné avec la toison d'or, navigua jusqu'à l'Isthme, où il consacra la nef Argos à Poséidon. Dion Chrysostome (Orat. 37, 15) ajoute que l'offrande du vaisseau au sanctuaire de Poséidon Isthmique fut accompagnée d'une inscription qui disait :

"Je suis la nef Argos. Jason me consacra au dieu, ayant vaincu aux jeux isthmiques et couronnée de pins de Némée".

On ne s'accorde pas sur la destinée finale de ce célèbre bateau, car tandis que Martial (Epigr. 7,19$)$ affirme qu'une partie du bateau était encore conservée à Rome comme une relique, Hygin $(F a b .14,33)$ dit que Athéna, constructrice du bateau, l'emporta au cercle des Astres.

Dans l'héraion même de Samos, il semble qu'à l'époque archaïque cette coutume de consacrer des bateaux existait déjà, si l'interprétation que l'on fait de l'usage d'un podium où l'on aurait déposé de telles offrandes, est juste. À Délos, on conserve le lieu où Antigone déposa le vaisseau amiral après le triomphe à Cos. Et encore en 31, Auguste offrit à l'Apollon d'Actium les vaisseaux de son triomphe et une colonne rostrata avec les éperons des navires ennemis.

Parfois, ce ne sont pas les vaisseaux eux-mêmes mais des statues allusives que l'on consacre. En ce sens, l'exemple le plus réputé est la célèbre Victoire de Samothrace, conservée au Louvre, qui faisait partie d'une triemiolia consacrée comme offrande par l'État rhodien ; mais on peut aussi rappeler le monument en forme de proue de vaisseau à Cyrène, les découvertes du sanctuaire d'Athéna lindienne à Rhodes ou celles du sanctuaire des Grands Dieux de Samothrace ; ces dieux à qui étaient consacrés les "mystères mineurs" avaient un lien très étroit avec la mer et avaient la réputation de protéger les naufragés.

Pour apaiser les dangers de la mer il y a des prières spécifiques, mais elles ne conviennent pas toujours au goût capricieux de la divinité. Baptiser le vaisseau d'un théonyme n'était pas même une garantie; mais il était fréquent de rechercher la complicité divine en appelant le bateau "Isis" ou "Minerve",

22 Bibl. I, 9, 27. 
comme celui qui paraît-il emmena Ovide en exil. Les inscriptions de la flotte de Mycènes nous permettent de savoir que, des neuf vaisseaux à quatre rangs de rames (tétrères), huit portent un théonyme. On plaçait souvent des statues de dieux à bord comme outils apotropaïques (telle est précisément la fonction de l'œil peint sur la proue, qui s'est perpétué jusqu'à nos jours). Plus fréquemment, on plaçait une représentation de la divinité tutélaire sur la proue, le parasemon. Mais on emportait aussi d'autres images; par exemple, le vaisseau de Lindos avait une statuette placée sous le siège du triérarque.

Même les corps de métier en rapport avec des activités maritimes se soumettaient à la tutelle d'une divinité ayant des pouvoirs sur la mer. En ce sens, depuis l'époque hellénistique, les dieux alexandrins Isis et Sérapis jouirent d'un prestige extraordinaire, à tel point que la fête qui inaugurait la saison nautique fut le navigium Isidis, dont le contenu nous a été transmis par Apulée au livre XI, 16, des Métamorphoses23. De nombreux sanctuaires isiaques étaient décorés de tableaux représentant des naufrages en signe de gratitude de la part des survivants envers la déesse bienfaisante24. De même, Stace, qui vécut tout au long du $\mathrm{I}^{\mathrm{er}}$ siècle en Campanie, région hospitalière pour les dieux égyptiens, dans le poème qu'il composa à l'occasion de l'embarquement à Puteoli de Metius Celer en direction de l'Orient, $\mathrm{n}^{\prime}$ oublie pas de confier le voyageur à Isis :

«Isis, jadis parquée dans les grottes de Phoronée, à présent reine de Pharos et déesse de l'Orient haletant, salue du bruit multiple de ton sistre le vaissaux de Maréotide; et ce jeune homme éminent, auquel le chef du Latium a confié ses étendards d'Orient avec le commandement des cohortes de Palestine, conduis-le toi-même d'une main bienveillante par les temples en fête, par tes ports sacrés, par tes villes ${ }^{25}$.

Lorsqu'un danger menaçait, on prenait les mesures correspondantes, non seulement à titre personnel, car le capitaine du vaisseau exigeait de même un comportement adéquat de la part de tout l'équipage et des passagers. Les sacrifices propitiatoires sont bien connus dans l'abondante littérature liée aux explorations maritimes. Mopsos induit ses compagnons de la nef Argos à réaliser des

23 Pour tout ce qui concerne ces fêtes et, en général, la vénération d'Isis comme protectrice des navigateurs, voir J. Alvar, Misterios.Religiones “orientales" en el Imperio Romano, Barcelone, 2001, 218.

24 Au sujet des tableaux consacrés par les fidèles favorisés: Tibulle I, 3, 27-28, qui démontre la continuité de la pratique, au moins, du $\mathrm{I}^{\mathrm{er}}$ au $\mathrm{II}^{\mathrm{e}}$ siècle av. J.-C. Nombre des lampes conservées avec une représentation d'Isis Pelagia, dominant la mer depuis un bateau (Ph. Bruneau, "Existe-t-il des tableaux d'Isis Pelagia?", BCH, 98, 1974, 333-381, spécialement 338), semblent répondre aussi au remerciement des navigateurs.

25 Stace, Silves III, 2, 101-107; T. I (Livres I-III) texte établi par Henri Frère et traduit par H.J. Isaac ( $3^{\text {è }}$ tirage, revu et corrigé par Claude Moussy), París, Belles Lettres, 1992. Cf. S. Montero, "Los dioses egipcios en Estacio", Habis, 10-11, 1979-1980, 241-253. 
libations et un sacrifice en l'honneur d'Apollon Sauveur des Bateaux (Apol. Rod., Arg. II, 927 ; l'épithète "Neeossoos" est aussi appropriée pour Artémis, ibidem, I, 570).

Mais l'anecdote la plus célèbre se rapportant aux attributions du capitaine du vaisseau en danger est probablement celle du Livre de Jonas (1, 4-15), qui, prétendant échapper à la volonté de Yahvé, ne fait au contraire qu'exacerber le dieu colérique de l'Ancien Testament:

Mais Yahvé lança sur la mer un vent violent, et il y eut grande tempête sur la mer, au point que le vaisseau menaçait de se briser. Les matelots prirent peur; ils crièrent chacun vers son dieu, et pour s'alléger, jetèrent à la mer la cargaison. Jonas cependant était descendu au fond du bateau ; il s'était couché et dormait profondément. Le chef de l'équipage s'approcha de lui et lui dit: “Qu'as-tu à dormir? Lève-toi, crie vers ton Dieu! Peut-être Dieu songera-t-il à nous et nous ne périrons pas."

Puis ils se dirent les uns aux autres: "Tirons donc au sort pour savoir de qui nous vient ce mal." Ils jetèrent les sorts et le sort tomba sur Jonas...

Les hommes furent saisis d'une grande crainte et ils lui dirent: “...Que te feronsnous pour que la mer s'apaise pour nous? ... Il leur répondit : Prenez-moi et jetez-moi à la mer, et la mer s'apaisera pour vous. Car, je le sais, c'est à cause de moi que cette violente tempête vous assaille."

Les hommes ramèrent pour gagner le rivage, mais en vain, car la mer se soulevait de plus en plus contre eux... Et, s'emparant de Jonas, ils le jetèrent à la mer, et la mer apaisa sa fureur..."

Comme on peut l'apprécier, quand les prières ne suffisent pas, on peut solliciter la pitié des dieux par des moyens plus drastiques. Encolpe, dans le Satiricon $(105,4)$ affirme :

“... pour satisfaire la divinité protectrice du navire, on décida de nous infliger à chacun quarante coups de fouet. Et, tout aussitôt, les matelots furieux s'emparent de nous, cordes en main, et tentent d'apaiser la divinité avec notre sang servile". (Les extraits du Satiricon correspondent à la traduction de Pierre Grimal, Paris, 1959).

Il est vrai que le dieu de l'Ancien Testament est démesuré dans sa cruauté, mais les dieux gréco-romains n'ont souvent rien à lui envier. Les raisons pour lesquelles les personnages du Satiricon reçoivent un châtiment importent peu ici ; ce que nous voudrions souligner c'est la certitude, toujours, que les malheurs proviennent d'un comportement personnel impie. Par conséquent, les sentiments religieux sont profondément ancrés dans la pensée des gens, déployant leurs ramifications jusqu'au plus profond de l'épiderme. La religion s'empare de la pensée et de l'action à un point insoupçonné. À bord du vaisseau il est indispensable d'éviter les actes impies et il est nécessaire de maintenir à distance quelque pollution que ce soit. Les excrétions, les taches, les résidus et flux 
corporels, tout ce qui constitue l'univers des tabous corporels a sa projection sur le bateau. Il est, bien sûr, interdit d'avoir des rapports sexuels à bord mais aussi de se couper les cheveux ou les ongles (Satiri., 104, 5). Ces impuretés polluent le vaisseau et irritent les dieux, en particulier ceux de la mer. Ceux-ci n'admettent d'autre offrande que la chevelure. (Cela dit, les besoins biologiques ne pouvant être contenus, il y a permissivité : sur un vase du VIII siècle on voit même représenté un matelot se soulageant depuis la barre du gouvernail d'un navire marchand, et je crois bien qu'un autre personnage accroupi est en train de faire de même sur l'éperon du vaisseau tandis qu'un autre pêche depuis le château de proue sur le fameux relief du temple d'Artémis "Ortia" de Sparte).

Achille Tatius (V, 16, 7-8) affirme en ce sens : "les bateaux doivent être nets de tout rapport amoureux, soit parce qu'ils sont sacrés, soit pour que personne ne s'adonne au plaisir dans des moments aussi dangereux".

Les personnages du Satiricon $(103,2)$, pour éviter d'être reconnus par ceux qui les poursuivent, décident de faire semblant d'être des esclaves fugitifs capturés par leur maître :

“La supercherie fut immédiatement réalisée; nous gagnâmes à la dérobée le bord du navire et nous tendîmes au barbier tête et sourcils pour qu'il les rase. Eumolpe nous couvrit le front à tous deux de lettres énormes et nous inscrivit largement sur toute la figure l'inscription habituelle des esclaves fugitifs. Mais il se trouva que l'un des passagers, penché sur le bordage, en train de soulager son estomac que dérangeait le mal de mer, remarqua sous la lune un barbier en train d'exercer son métier à cette heure indue, et, maudissant ce présage, qui évoquait le vœu suprême des naufragés, il se remit au lit. Nous, faisant semblant de ne pas entendre les malédictions du passager malade, nous retombons dans nos soucis habituels, et, nous installant en silence, nous passons le reste de la nuit dans un mauvais sommeil".

La découverte de la supercherie est la cause des quarante coups de fouet auxquels nous faisions allusion plus haut. Ainsi, dans des situations extrêmes, lorsque l'habileté du pilote s'est avérée inutile, les prières inefficaces et que l'on tente d'apaiser sans succès les dieux avec des boucs émissaires, alors on fait les offrandes les plus insolites et des promesses que, lorsque tout se termine, on souhaiterait ne pas avoir formulé. Les plus intrépides osent les éviter, mais la plupart s'y tiennent scrupuleusement. Tous les efforts sont destinés à éviter le destin final provoqué par une tempête implacable. Et lorsque tout est perdu, chacun donne libre cours à son dernier désir. L'une des descriptions les plus saisissantes des instants précédant un naufrage provient du Satiricon. Encolpe le raconte à la première personne : 
“... la mer devint houleuse, des nuées s'amassèrent de toutes parts, obscurcissant le jour. Les matelots courent en tous sens à leur poste, dans une grande agitation, et larguent les voiles devant la tempête. Mais le vent ne soufflait pas dans une direction constante, et le pilote ne savait pas quel cap prendre... Comprenant que le danger devenait extrême, Lichas se jeta en tremblant à mes pieds et, me tendant des mains suppliantes: "Toi, Encolpe, dit-il, vient à notre secours dans ce péril, rends au navire le vêtement divin et le sistre..."

Il criait encore lorsqu'un coup de vent le fit basculer dans la mer; on le vit remonter puis la tempête le fit tournoyer sur le gouffre menaçant et l'engloutit... Moi, serrant Giton contre moi, je pleurai à grand bruit, disant: "Qu'avons-nous donc fait aux dieux pour qu'ils nous unissent seulement dans la mort? Mais la Fortune, impitoyable, ne nous le permet pas ... voici que la mer agitée va rompre l'étreinte de deux amants. Aussi, si tu as vraiment aimé Encolpe, embrasse-le, tant que tu le peux, et arrache cette dernière satisfaction aux destins qui se hâtent vers leur accomplissement".

En m'entendant parler de la sorte, Giton quitta son vêtement, se couvrit de ma tunique et tendit son visage vers mes baisers. Et, pour que le flot, dans sa cruauté, ne pût nous séparer dans cette étreinte, il nous entoura tous deux de sa ceinture: "Au moins, dit-il, la mer nous portera plus longtemps unis de la sorte, et, si elle veut bien être assez miséricordieuse pour nous déposer sur le même rivage, ou bien, peut-être, dans un mouvement de pitié banale, quelque passant déposera-t-il sur nous des pierres, ou bien, dernière faveur des flots en courroux, le sable inconscient nous enterrera-t-il." J'accepte ce lien ultime, et, comme déposé sur le lit funèbre, j'attends une mort que je ne redoute plus" (114, 1-12).

Le naufrage a naturellement lieu mais les amants se sauvent, car nous ne sommes pas dans Titanic. C'est alors l'occasion de satisfaire les promesses réalisées dans les moments d'angoisse. Arrivés au port, ces mêmes matelots qui peu avant étaient morts de peur, deviennent des charlatans bravaches qui racontent à la taverne leurs fantaisies passionnées, même la tête rasée, comme le rappelle Juvénal dans sa Satire 12, 81-82: Tunc stagnante sinu, gudent ibi vertice raso / Garrula securi narrare pericula nautae. Les sceptiques peuvent se rassurer avec une autre perspective léguée par l'Antiquité elle-même. Cicéron raconte (Nat. Deor. III, 37, 39) une anecdote sur Diagoras de Mélos, selon laquelle un ami lui aurait demandé comment il était possible qu'il ne crût pas au fait que les dieux protégeaient les hommes alors que le sanctuaire des Dieux de Samothrace était rempli de tablettes votives peintes, offertes par des personnes sauvées d'un naufrage, ce à quoi Diagoras répondit avec audace que la raison de son critère s'appuyait sur le fait que les noyés ne peuvent pas peindre.

Mais les fanfaronnades n'empêchent pas que les sanctuaires se remplissent de tableaux représentant les naufrages et l'intervention miraculeuse de la divinité salvatrice, ainsi que d'ex-votos de toutes sortes qui manifestent de la 
gratitude des survivants. Il est évident que le bateau, ses parties ou sa miniaturisation sont un thème récurrent dans les ex-votos offerts par les gens de la mer. Le vaisseau comme véhicule, la rame comme moteur, le gouvernail comme guide ou l'ancre comme frein, constituent des allégories appropriées pour les situations à risque où l'intervention divine est sollicitée. Les reproductions d'embarcations conservées sont de natures très diverses: lampes en forme de bateau, représentation sur des reliefs votifs, des peintures, des tablettes, au graphite, en céramique, en orfèvrerie, et sous toutes les formes pouvant se présenter à l'artiste. La difficulté étant de pouvoir déterminer s'il s'agit d'une manifestation religieuse ou d'une simple expression artistique.

Parfois, l'offrande est constituée par l'une des parties ou des ustensiles du bateau. Certes, un des éléments les plus caractéristiques est la rame, qui apparaît souvent comme ex-voto. Cependant, nous voudrions maintenant faire allusion à un élément légendaire en rapport avec la rame, qui a pour point de départ l'Odyssée et qui débouche sur une série de petites histoires que, d'une façon ou d'une autre, nous pourrions regrouper sous le nom de conte du marin et de la rame. En effet, dans 1'Odyssée (XI, 121-134; XXIII, 267-284) on rapporte une curieuse histoire relative à l'offrande d'une rame. Pour apaiser la colère de Poséidon, Ulysse doit réaliser une offrande expiatoire en suivant la prophétie de Tirésias:

“... il faudrait repartir avec ta bonne rame à l'épaule et marcher, tant et tant qu'à la fin tu rencontres des gens qui ignorent la mer et, ne mêlant jamais de sel aux mets qu'ils mangent, ignorent les vaisseaux aux joues de vermillon et les rames polies, ces ailes des navires ... le jour qu'en te croisant, un autre voyageur demanderait pourquoi, sur ta brillante épaule, est cette pelle à grains, c'est là qu'il te faudrait planter ta bonne rame et faire à Poséidon le parfait sacrifice d'un bélier, d'un taureau et d'un verrat de taille à couvrir une truie ; tu reviendrais ensuite offrir en ton logis la complète série des saintes hécatombes à tous les Immortels, maîtres des champs du ciel; puis la mer t'enverrait la plus douce des morts". (Trad. Victor Bérard, Paris, 1955).

William F. Hansen ${ }^{26}$ a réuni 26 histoires différentes du "Marin et de la rame" dispersées dans toute la Grèce, la Grande Bretagne et l'Amérique, datant des XIXe et XX $X^{\mathrm{e}}$ siècles. À l'exception de quelques variations dans le récit ou les personnages - ce peut être Saint Elie ou Saint Nicolas en Grèce, ou un navigateur anonyme dans la tradition anglo-saxonne - tous racontent à grands traits qu'un marin, fatigué de la vie en mer, chargea sa rame sur son épaule et commença à

26 "Odysseus and the Oar: A Folkloric Approach", Approaches to Greek Myth, L. Edmunds éd., Baltimore-Londres, 1990, 241-272.

DHA supplément 1, 2005 
marcher (dans une autre version c'est l'ancre qu'il porte). Il rencontra d'abord un homme à qui il demanda s'il savait ce qu'il portait sur l'épaule, lequel répondit correctement une rame. Il continua sa marche jusqu'à ce qu'il arrive dans un lieu éloigné de la mer et il demanda à un autre homme s'il pouvait lui dire le nom de l'instrument qu'il portait à l'épaule. Comme cette personne sut seulement lui répondre qu'il s'agissait d'un bout de bois, il comprit alors qu'il était suffisamment loin de la côte, il planta sa rame dans la terre et s'installa définitivement sur ce lieu. Lorsque le récit a un fondement hagiographique il débute par un message apostolique et il se termine sur la fondation d'une église, à l'endroit où la rame est restée plantée.

Le récit de $\mathrm{l}^{\prime} \mathrm{O}$ dyssée a un caractère religieux, car c'est le sacrifice expiatoire nécessaire que Poséidon réclame à Ulysse pour que celui-ci puisse revenir chez lui. La mission consiste à remettre sa rame au dieu de la mer en un lieu où les habitants ignorent tout de la navigation et où, par conséquent, Poséidon ne fait pas l'objet d'un culte. Le sacrifice d'animaux constitue le début d'un culte là où auparavant le dieu n'était pas vénéré et c'est ainsi que les scholiastes de l'Odyssée interprètent la prophétie de Tirésias. C'est-à-dire que l'on peut abandonner la mer comme moyen de subsistance mais cela implique une offrande spéciale au dieu de la mer pour ne pas l'offenser et, dans ce but, rien de mieux que de diffuser son culte au moyen de copieuses offrandes sur de nouveaux territoires.

Mais la navigation n'est pas la seule à dépendre de l'assistance divine. $\mathrm{D}^{\prime}$ autres gens qui vivent de la mer, comme les pêcheurs, sont constamment en contact avec les dieux pour parvenir à survivre. Le produit de la pêche est, en contrepartie, une offrande appropriée pour les dieux bienfaisants. Comme exemple, je rappellerai un passage de Pausanias $(X, 9,3-4)$ où il raconte comment, à Corcyre, un taureau mugissait sur le rivage, ce qui entraîna la descente d'un berger sur la côte pour voir ce qui s'y passait et il trouva des thons en quantité. Les Corcyriens, qui n'arrivaient pas à en pêcher, consultèrent l'oracle de Delphes et sacrifièrent le taureau à Poséidon. Après le sacrifice ils réussirent à capturer les thons, et l'offrande qu'ils firent à Olympe et à Delphes représenta la dixième partie de la pêche. Les Corcyriens consacrèrent en plus un taureau en bronze à Apollon, à Delphes, dont la base fut découverte lors de fouilles archéologiques.

En rapport avec la dévotion consacrée à Apollon par les pêcheurs, Élien nous fournit une curieuse information (NA. 12.1 ; Plut. Mor. 976 c) selon laquelle, dans la baie de Myra, sur la côte lycienne, il y avait une fontaine et un temple consacrés à Apollon. Là-bas, le prêtre jetait aux poissons la chair des vaches sacrifiées. Si les poissons dévoraient la viande, c'était signe de bon augure; 
en revanche, s'ils rejetaient la viande sur la terre avec leur queue, c'était l'annonce de la colère divine. Les prêtres étaient aussi capables de prédire ce qui allait advenir en vertu des espèces qui venaient consommer la chair des victimes (Polycharm. Ap. Ath. VIII, 333 d-e). Cet Apollon protecteur des pêcheurs recevait ses récompenses. À Athènes il percevait un revenu fixe sur les pêcheries de thon d'un dème de la tribu Cécropide ; à Délos, il avait le monopole de la pêche du murex, dont on obtient la pourpre, et en de nombreux endroits, il était bénéficiaire d'un impôt sur la totalité des poissons capturés ${ }^{27}$.

Apollon lui-même est associé à un autre type d'adoration en sa qualité d'ami des navigateurs, sous l'épithète "Delphinios". L'origine de ce vocable est très ancienne, elle remonte à l'époque crétoise, mais on ne connaît pas de premiers sanctuaires spécifiques avant le VIII ${ }^{\mathrm{e}}$ siècle av. J.-C. Ce dieu-ci annonce des voyages, des changements de résidence favorables. Le dauphin se présente comme bienfaiteur des marins et de tous ceux qui se voient affectés par les avatars de la mer. C'est à eux tous que l'on devrait les nombreuses figurines de dauphin offertes comme ex-votos. Mais il n'est pas toujours facile d'identifier la divinité à qui est offert le dauphin, car il peut s'agir parfois de Mélicerte-Palémon. Celui-ci était le plus jeune fils de Ino et de Athamas. Héra insuffla la folie à ses parents qui lui donnèrent la mort. Selon certaines versions, ils le lancèrent dans un chaudron d'eau bouillante. Après avoir récupéré la raison, Ino prit son fils dans ses bras et se jeta à la mer depuis la roche Moluride. Le corps de l'enfant fut recueilli par un dauphin qui le déposa sur l'Isthme de Corinthe. C'est là que Sisyphe le trouva et instaura en son honneur les jeux Isthmiques.

Mais les navigateurs reconnaissants n'offraient pas uniquement des exvotos ou des dons pécuniers. L'érection de temples est fréquente, coutume qui apparaît déjà dans l'Odyssée, lorsque les compagnons du héros tuent les vaches de Hélios sur l'île du Trident et pour éviter que la prophétie de Tirésias ne s'accomplisse, ils promettent au dieu de lui ériger un sanctuaire s'il les rend sains et saufs à leur patrie. Zeus venge l'outrage fait à Hélios en provoquant le naufrage où meurent tous les compagnons d'Ulysse, et il est le seul à pouvoir se sauver (Od. XI, 106-115, prophétie de Tirésias ; XII, 319-450, mort des vaches et naufrage). D'autre part, Pindare ( $P$. IV, 203-209) indique que les Argonautes consacrèrent un tememos à Poséidon à l'entrée du Pont, non pas Euxin, mais Áxeinos (Inhospitalier), vu le danger que représentaient les Roches Cyanées, dont la particularité est qu'elles bougeaient à cause de la houle et se heurtaient entre

27 Toute l'information à ce sujet est recueillie par Romero Recio, Cultos marítimos..., 73. 
elles, de telle manière qu'elles écrasaient les vaisseaux qui prétendaient entrer dans la mer Noire.

Aphrodite est de même honorée avec des sanctuaires par les marins reconnaissants. Énée lui consacre plusieurs sanctuaires depuis sa sortie de Troie jusqu'à son arrivée en Italie (D.H. I, 50, 1, 4;51, 2 ; 53, 1). Mais la fondation de temples n'est pas exclusive des temps mythiques et se répète au contraire constamment: les Athéniens érigèrent un sanctuaire à Borée, qui les avait aidés à vaincre Xerxès en lui envoyant une tempête, et Thourioi en fit de même lorsqu'elle fut miraculeusement sauvée par le même vent face à l'attaque de Denys de Syracuse.

Parfois c'est la concurrence entre les dieux qui provoque des vénérations déterminées. On connaît bien le conflit qui oppose Poséidon à Athéna pour le patronage d'Athènes, mais le cas d'Argos est moins connu, dont les habitants construisirent un sanctuaire à Poséidon "Prosclitos" ("celui de l'inondation"), pour remercier la divinité qui, auparavant, leur avait envoyé un terrible châtiment (Paus. II, 22, 4). Le dieu avait inondé d'eau de mer la région parce qu'Inachus et ses juges avaient décidé que la terre appartenait à Héra et non pas à Poséidon; peut-être cette fois-ci la colère divine était-elle justifiée, car selon d'autres sources, nous savons que le dieu de la mer avait révélé aux Argiens la localisation de quelques sources d'eau potable.

Il est évident que les dieux ne pardonnent pas l'impiété (asebeia) des dévôts et qu'ils exigent que les cérémonies soient strictement accomplies. Ménélas embarqua sans avoir réalisé les sacrifices habituels et il fut pour cela châtié (Od. IV, 351-353). Un amiral macédonien, Dicéarque, ne réalisait pas les sacrifices traditionnels au moment de débarquer, mais il érigeait au contraire des autels à ses divinités particulières, l'Impiété et l'Injustice, ce pour quoi il reçut son châtiment: il mourut après avoir été flagellé et torturé sur le chevalet (Polib. XVIII, 54, 8). Denys de Syracuse fut lui aussi châtié pour s'être vanté d'avoir bien navigué après avoir commis un sacrilège (Val. Max. I, 3). Le cas d'Ajax est lui aussi exemplaire en ce sens, lequel sortit Cassandre du temple d'Athéna où elle s'était réfugiée. Poséidon déchaîna une tempête qui fit naufrager les vaisseaux, mais le héros se sauva. Il se vantait d'avoir surmonté la colère divine, ce qui irrita d'autant plus le dieu de la mer qui brisa de son trident le rocher qui lui avait permis de se sauver (Od. IV, 499-511). Hyppase de Métaponte fut châtié par un naufrage pour avoir révélé un secret de géométrie découvert dans son école, d'après le récit de Iamblichus (VP XVIII, 34). 
Les sacrifices pour purifier les vaisseaux furent aussi fréquents dans l'Antiquité ; la description la plus saisissante, nous la trouvons chez Appien (B.C. $\mathrm{V}, 96)$ :

"On érige des autels au bord de la mer et la foule se rassemble autour d'eux, à bord des navires, dans un profond silence. Les prêtres réalisent les sacrifices debout, en face de la mer et par trois fois ils apportent les victimes du sacrifice sur des barques autour de la flotte; ils se font accompagner des généraux tandis qu'ils profèrent des imprécations aux dieux pour diriger les mauvais augures vers les victimes expiatoires et non vers la flotte. Après, en les morcelant, ils en jettent une partie à la mer et posent l'autre sur les autels pour les brûler tandis que le peuple les accompagne de leurs chants".

Virgile reprend une image semblable (Aen. V, 772-776), lorsque Énée, avant de reprendre la navigation, sacrifie trois veaux en l'honneur du héros Éryx et un mouton aux tempêtes et, par la suite, il lance à la mer les entrailles des victimes depuis la proue en versant quelques liquides.

Les sacrifices étaient réalisés comme démonstration de piété envers n'importe quelle divinité. Poséidon en est logiquement l'un des principaux destinataires et l'on a pour coutume de lui sacrifier un taureau, à tel point qu'il reçoit l'épithète de "Taureos". Les exemples sont fort nombreux, il suffit de rappeler qu'au milieu de la traversée de l'Hellespont, Alexandre trancha la gorge d'un taureau en l'honneur de Poséidon et versa une libation dans la mer avec une coupe en argent en l'honneur des Néréides. En tant que divinité suprême, Zeus est aussi le destinataire de sacrifices fréquents ainsi que Apollon et les Dioscures, et Aphrodite (qui apprécie particulièrement les chèvres - comme l'indique une scholie des Dialogues des courtisanes de Lucain - par leurs qualités aphrodisiaques et leur intempérance sexuelle), ainsi que Hermès ou les Vents. Il peut paraître plus surprenant que l'on sacrifie aux morts pour obtenir leur intervention positive ou pour éviter leur intrusion pendant la navigation: Achille exige le sacrifice de Polyxène auprès de son sépulcre sous menace de provoquer une tempête et les Argonautes réalisent des libations et des offrandes en l'honneur de Sthénélus Actoridien et d'Apollon sur la tombe du premier, lequel était mort près d'un promontoire après avoir accompagné Héraclès dans la guerre contre les Amazones. Jason offre les amarres de son vaisseau à l'embouchure du Phase en l'honneur des âmes des héros morts, des dieux et de la Terre, pour qu'ils soient des "protecteurs généreux" 28 .

28 Romero Recio, "Conflictos entre la religiosidad...", 45. 
Les biens personnels pouvaient aussi constituer une offrande appropriée pour apaiser les dieux. Dans l'Anthologie Palatine on trouve des offrandes telles que le bonnet d'un pêcheur, le chapeau d'un voyageur, le manteau d'un navigateur sauvé d'une tempête et avec la collection de témoignages provenant d'autres sources, on peut conclure que des vêtements en tout genre étaient appropriés pour la cause ${ }^{29}$. Offrir des vêtements implique la disposition de celui qui fait l'offrande face à la divinité, le don absolu jusqu'à la nudité. L'offrande du vêtement, comme celle de la chevelure, implique une proximité évidente entre le naufragé et l'esclave, de telle manière qu'elle suppose un renoncement implicite à la liberté et une soumission totale à la divinité protectrice.

Les témoignages littéraires nous permettent de savoir que la peau de certains animaux avait des pouvoirs magiques. Plutarque (mor. 664c) indique que la peau des phoques et des hyènes maintenait les rayons éloignés du mât. Dans $1^{\prime}$ Odyssée (X,19-26) les vents sont enfermés dans une outre fabriquée avec de la peau de bœuf pour éviter leur néfaste intervention pendant la traversée. Une famille de Corinthe, les Anemokoitai, possédait la qualité magique d'exercer le contrôle sur les vents ${ }^{30}$. Il est évident que les arts divinatoires et la magie étaient présents dans la vie des marins ou dans celle de ceux qui avaient à fréquenter la mer. À Corinthe, d'après le témoignage des Métamorphoses (II, 12, 35) d'Apulée, il y avait un devin qui gagnait sa vie en faisant des prédictions, entre autres, sur les dates qui assureraient un heureux voyage par voie terrestre ou maritime. Apulée lui-même (Mét. III, 17, 4-5) parle d'une sorcière qui utilisait des restes de navires naufragés parmi les engins qui lui servaient à faire ses conjurations.

Pour apaiser les malheurs en mer on allait jusqu'à réaliser des sacrifices humains. Rappelons les cas mentionnés plus haut de Jonas et d'Iphigénie. D'une façon ou d'une autre la coutume persista et, comme pour émuler la fameuse histoire biblique de Jonas, au VII ${ }^{\mathrm{e}}$ siècle ap. J.-C., Ioannes Moschos raconte $l^{\prime}$ histoire d'un navire d'Alexandrie, immobilisé par le calme plat ${ }^{31}$. Le capitaine se tourna vers Dieu pour en demander la raison. Une voix répondit: "jette Marie à la mer". Parmi les passagers se trouvait une femme du nom de Marie qui se vit obligée de confesser sa faute. Veuve avec deux enfants, elle était devenue la mâ̂tresse d'un soldat qui refusait de se marier avec elle car il ne voulait pas être

Romero Recio, Cultos marítimos..., 109.

Sur ce thème et les exemples qui sont montrés ici, Romero Recio, Cultos marítimos..., 99.

Ioannes Moschos, Pratum spirituale, Bibliotheca Hagiographica Graeca 1450 ib, (éd. F. Halkin,

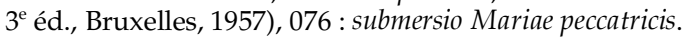


responsable des deux enfants. À cause de cela, la femme avait tué ses propres enfants, mais son amant, horrifié par le terrible crime, l'avait abandonnée. Le sacrifice n'eut pas lieu comme l'avait préconisé la voix divine, car le capitaine, ayant pitié de la femme, ne la jeta pas à la mer mais l'abandonna dans une barque. L'embarcation sombra soudain avec la femme et au même moment, le vent se mit à souffler, le navire pouvant ainsi reprendre sa route sous les yeux encore ébahis des témoins.

D'après l'histoire rapportée par Hérodote (IV, 103), les Taures de Scythie avait la coutume de sacrifier les survivants d'un naufrage en l'honneur d'Artémis-Iphigénie, en suivant toujours le même rituel :

“après avoir consacré la victime, ils la frappent à la tête avec une massue. D'après les uns, ils précipitent le corps du haut en bas du rocher (car le temple est bâti sur un rocher escarpé), et fixent la tête à une croix ; d'autres, d'accord avec les précédents pour ce qui est de la tête, prétendent que le corps n'est pas précipité du rocher, mais enfoui dans la terre. À ce que disent les Taures eux-mêmes, la divinité à laquelle ils offrent ces sacrifices serait Iphigénie fille d'Agamemnon"32.

Les nouvelles se rapportant à des épisodes sur des sacrifices humains sont déjà soumises à une forme culturelle, de telle façon qu'ils n'apparaissent pas comme des sacrifices mais comme des exécutions. On a donc l'impression qu'il y a eu transfert depuis la sphère du sacrifice rituel jusqu'à celle de l'exécution personnelle, dans un processus d'adaptation culturelle.

"Seule la divinité à qui l'on consacrait et les circonstances de la mort nous permettent de percevoir que l'origine de ces peines pourrait se trouver dans un cadre religieux en rapport avec la mer et la navigation"33.

Nous concluons ainsi ce parcours où l'on prétendait montrer comment la religiosité des navigateurs était déterminée non seulement par le milieu où se déroulaient leurs activités socio-économiques, mais aussi par l'ensemble des croyances propres à la communauté à laquelle ils appartenaient. En effet, la presque totalité des dieux du panthéon gréco-romain possédait une facette qui les mettait en rapport avec la mer, de là ils auraient eu un culte spécifique de la part des navigateurs. La proximité de ces cultes avec la religion de la cité faisait que l'État lui-même régla les rythmes et les modalités de cette religiosité spécifique. Mais l'imaginaire des navigateurs se développa d'une façon spéciale à cause de l'espace physique dans lequel ils vivaient. C'est dans ce milieu, dépositaire de

32 Hérodote, Histoires, Livre IV. Texte établi et traduit par Ph.-E. Legrand, Paris, Belles Lettres, 1949.

33 Romero Recio, Cultos marítimos..., 105. 
nombre de leurs pollutions, qu'ils installent des êtres merveilleux ou répugnants à qui l'on attribue la responsabilité des avatars quotidiens. Une fois construit cet univers fantasmatique, il faut inventer les instruments qui serviront à l'affronter. La cité fournit des moyens classiques, comme l'érection d'autels ou de sanctuaires. La religiosité traditionnelle offre des mécanismes de réussite à travers la prière, les offrandes, les sacrifices. Mais tout ceci apparaît comme insuffisant et laisse la place à d'autres pratiques plus éloignées des rituels propres à la religion civique. S'ouvre alors un immense territoire dont les pratiques déconcertantes auraient laissé bouche bée les dieux eux-mêmes, s'ils avaient existé.

Traduction Cristina Abril Soubagné 